- Oral surgery can aid orthodontics in managing ectopic teeth in patients with hypodontia

- Oral surgery is useful in managing hypodontia as it can move teeth between the jaws

- Oral surgery can aid prosthodontic management of young people with hypodontia

\title{
Interdisciplinary management of hypodontia: oral surgery
}

\author{
J. G. Meechan, ${ }^{1}$ N. E. Carter, ${ }_{1}^{2}$ T. J. Gillgrass, ${ }_{1}^{3}$ R. S. Hobson, ${ }_{1}^{4}$ N. J. Jepson, ${ }^{5}$ F. S. Nohl, ${ }^{6}$ and J. H. Nunn, ${ }^{7}$
}

\begin{abstract}
This paper describes the role of oral surgery in the management of children and adolescents with hypodontia. Surgical intervention can be used as an aid to orthodontic and prosthodontic treatment. In some cases surgery can supersede orthodontics as transplantation makes it possible to move teeth between the jaws.
\end{abstract}

\section{THE INTERDISCIPLINARY MANAGEMENT OF HYPODONTIA: \\ 1. Paediatric dentistry \\ 2. Restorative dentistry \\ 3. Orthodontics \\ 4. Oral surgery \\ 5. The relationship between an interdisciplinary team and the general dental practitioner}

1Senior Lecturer/Honorary Consultant in Oral Surgery ${ }^{2}$ Consultant in Orthodontics, ${ }^{4}$ Senior Lecturer/Honorary Consultant in Orthodontics ${ }^{5}$ Senior Lecturer/Honorary Consultant in Restorative Dentistry, ${ }^{6}$ Consultant in Restorative Dentistry, Department of Restorative Dentistry, School of Dental Sciences, University of Newcastle upon Tyne and Newcastle Dental Hospital; ${ }^{7}$ Professor and Head of Department, Department of Public and Child Dental Health, Dublin Dental Hospital, Ireland: ${ }^{3}$ Consultant in Orthodontics, Department of Orthodontics, Edinburgh Dental Institute, Scotland Correspondence to: Dr J. G. Meechan, School of Dental Sciences, University of Newcastle upon Tyne, Framlington Place, Newcastle upon Tyne, England NE2 4BW E-mail :J.G.Meechan@ncl.ac.uk

\section{Refereed Paper}

๑ British Dental Journal 2003; 194: 423-427
An oral surgeon may seem an unlikely member of a hypodontia team. However this specialist can play an integral role in the management of young people with missing teeth. The obvious function might appear to be in the placement of implants. However, in the management of the young patient with hypodontia this may not be the most appropriate approach and surgical treatment offers alternative strategies.

In the management of hypodontia surgery can:

- Aid orthodontics

- Supersede orthodontics

- Aid prosthodontics.

\section{SURGERY AS AN AID TO ORTHODONTICS}

A combined surgical/orthodontic approach at the treatment planning stage is of benefit to many patients. The surgeon can aid the orthodontist by offering the following services:

- Surgical exposure of unerupted teeth

- Attachment of orthodontic appliances to unerupted teeth.

\section{Surgical exposure of unerupted teeth}

The exposure of unerupted teeth can vary in complexity from a simple soft tissue procedure readily achieved under local anaesthesia to more involved operations which may necessitate general anaesthesia as an adjunct.

When considering the exposure of unerupted teeth the following factors are important:
- Accurate localisation of the tooth

- The type of soft tissue overlying the crown of the tooth.

\section{Localisation of the tooth}

Clinical and radiographic interpretation are essential in this regard. Clinical examination includes palpation and appreciation of the coronal/root inclination of neighbouring erupted teeth. Radiographic interpretation involves taking more than one radiograph as the use of parallax is required to accurately locate the unerupted tooth in the labio-lingual plane. Although it is sometimes possible to determine labio-lingual position with a single panoramic view (palatally placed teeth appear larger than those in the arch), alternative suitable radiographs include parallax periapical films or an orthopantomogram plus an occlusal film.

The type of soft tissue overlying the crown of the tooth

Is it attached or unattached gingiva? When the crown of the tooth is covered by attached gingiva (for example, palatal mucosa), simple excision of the overlying gingiva including any bone removal, where necessary, is sufficient. The gingival defect may be packed with either ribbon gauze soaked in Whitehead's varnish or a periodontal dressing such as Coe-Pak (Fig. 1) can be sutured in place or added to a removable appliance. The authors' preference is to use Coe-Pak as it appears to produce a better tissue response. Ideally this is added to a removable appliance. In addition to avoiding the use of sutures (an 


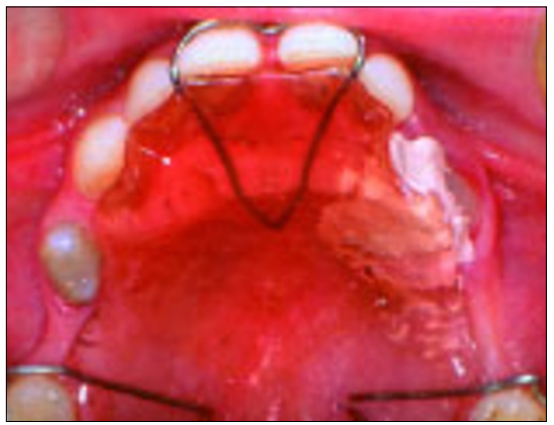

Fig. 1 Coe-Pak loaded on to an upper removable appliance and used as a dressing over a surgically-exposed upper premolar tooth

advantage as far as the patient is concerned) an appliance, particularly in the upper jaw, can provide excellent haemostasis post-operatively. This is important since the sacrifice of palatal tissue overlying an unerupted maxillary canine often produces copious bleeding because the incision is made at right angles to the direction of the greater palatine artery. The pack should be removed between 7 and 10 days after surgery. After pack removal the patient is advised to scrupulously brush the area around the exposed tooth. This is essential to prevent early overgrowth of the adjacent gingivae.

When the crown of the tooth is covered by unattached gingiva, there are two options. The first is to approach the tooth through attached gingiva and, following any bone removal, reposition the flap apically to its original location. The alternative is to attach an orthodontic appliance to the unerupted tooth (see later). The method of choice is attachment and orthodontic traction to encourage eruption through attached gingiva as this produces an optimal gingival contour. ${ }^{1}$

Occasionally overlying primary teeth must be removed to aid exposure. This is usually a straightforward procedure. An exception is when the primary tooth has 'submerged'. In such circumstances the infra-occluded tooth sometimes has to be removed by a surgical approach.

\section{Attachment of orthodontic appliances to unerupted teeth}

Various attachments can be placed on teeth which have been surgically exposed. These include:

- Gold chains

- Stainless steel ligatures

- Magnets.

These devices are attached to teeth in the following circumstances:

- When the tooth is lying beneath reflected mucosa

- When the tooth is deeply impacted and maintenance of a satisfactory, patent eruptive channel is not possible after pack removal.
When performing these procedures a mucoperiosteal flap is raised. When a chain or ligature is to be attached, part of the incision is made over the normal eruptive path of the tooth. After bone removal, the surgical area is maintained in as dry a state as possible. The attachment is retained using an acid etch composite bonding technique with orthodontic composite in exactly the same method employed in erupted teeth (Fig. 2). A material which is less susceptible to moisture and blood contamination should be used. $^{2}$ The use of a wire ligature around the tooth is best avoided. ${ }^{3}$ Once the appliance has been bonded the mucoperiosteal flap is replaced in the original position. If a chain or wire has been attached this exits the wound through the point where eruption is intended.

When gold chains are used the free-end of the chain may be attached to an erupted tooth with a composite bond or simply sutured to mucosa prior to attachment to an orthodontic appliance (Fig. 3).

When a magnet is employed (Fig. 4) the magnet of the opposite pole is incorporated into a removable orthodontic appliance or prosthesis, prior to the surgery. The surgical approach is identical to that described for attachment of a gold chain.

\section{SURGERY SUPERSEDES ORTHODONTICS}

Surgery can outperform orthodontic movement in a number of ways. Firstly, it can move teeth resistant to other means and it can move teeth quickly. Ectopic teeth unsuited to orthodontic movement in the hypodontia patient can be moved rapidly by transplantation. In addition, surgery allows movement of teeth between the

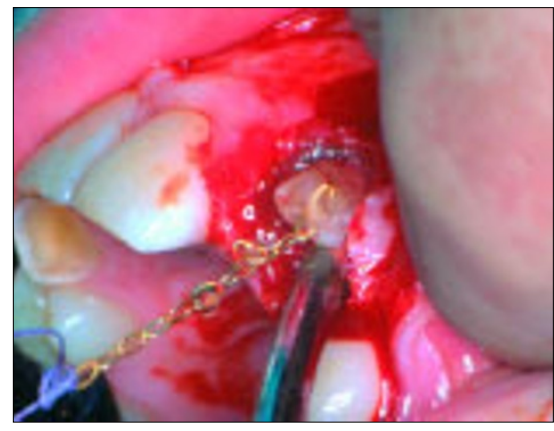

Fig. 2 A gold chain bonded on to an unerupted canine to aid eruption through attached gingiva

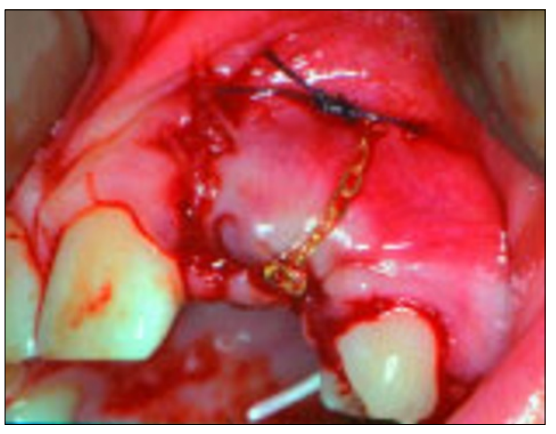

Fig. 3 The free-end of a gold chain which is bonded to an unerupted canine attached to gingiva with a suture 

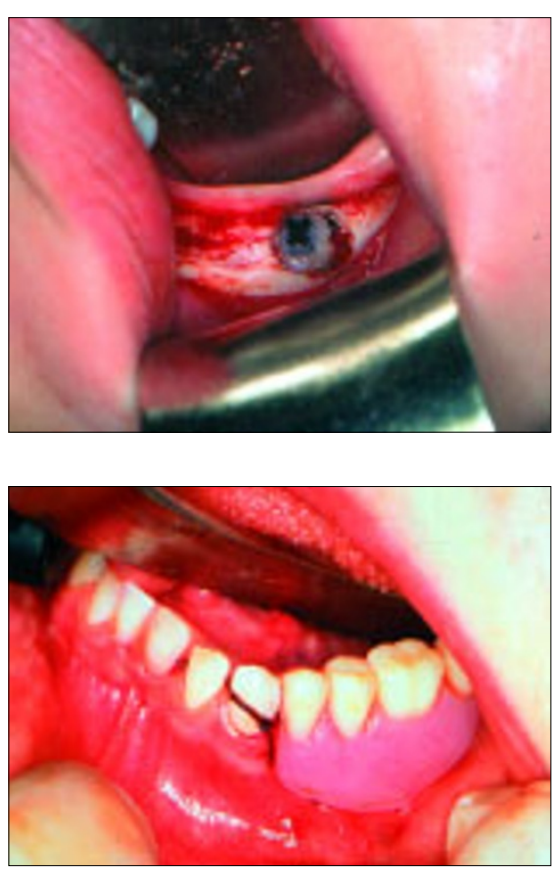

Fig. 4 The use of magnets: a) A magnet attached to an unerupted mandibular premolar in a child with hypodontia. b) The opposite pole of the magnet is contained in the lower partial denture (Fig. 4a is reproduced by permission of Oxford University Press)

jaws. Thus transplantation can be used to correct hypodontia in one jaw when redundant teeth are present in the opposite jaw or the other side of the arch. A number of teeth are suitable for transplantation in ectopic sites. The lower second premolar is a particularly versatile tooth and is especially useful in replacing upper anterior teeth (Fig. 5). ${ }^{4,5}$ Canines and small upper third molars are also amenable to transplantation to the upper incisor region. The success of transplantation is very good: 5 year survival is reported to be greater than $98 \%$ and 10 year survival at over $87 \% .^{6}$

A number of factors must be taken into account when considering tooth transplantation. The ideal time to remove the tooth is before root formation is complete. Ideally two-thirds to three-quarters root development should have occurred. The more root formation the greater the chances of root resorption and the poorer pulpal healing. ${ }^{7,8}$ A single prophylactic dose of an appropriate antibiotic such as amoxicillin should be given to cover the operative procedure. This may be given orally one hour pre-operatively or intravenously during the procedure. The aim is to ensure adequate anti-microbial protection of the initial blood clot. When removing the tooth the usual rules about application of the forceps beaks to the root surface do not apply. It is imperative that the root surface (principally the periodontal ligament) is subject to no or minimal trauma during the extraction. This may be achieved by running a scalpel fitted with a number 11 blade round the marginal gingiva. The root of the tooth can then be protected by placing a cotton dental roll over the crown (Fig. 6) and then applying the forceps to the crown to effect removal. In addition to protecting the periodontal ligament the use of a cotton roll reduces the chances of enamel fractures which can readily occur when teeth are removed by applying forceps to the crown. The recipient site should be prepared following mobilisation of the donor tooth. It is unwise to prepare the recipient site earlier in case the tooth scheduled for transplantation cannot be satisfactorily removed. Modification of the donor site may be required in order to achieve a close fit. It is unwise to repeatedly insert and remove the donor tooth into the prepared socket. The longer the time the donor tooth spends in an extra-alveolar environment the poorer is the post-operative healing. ${ }^{7,8}$ Either an artificial root or a similar unwanted tooth extracted at the same visit (for example the contralateral tooth) should be used as a template if possible. Where there is an excellent fit of the transplanted tooth in the recipient socket it can be held in position by running a suture over the incisal/occlusal surface from buccal to lingual gingiva. If there is not a tight adaptation the transplanted tooth is stabilised by attaching it to adjacent teeth with orthodontic wire, usually nickel-titanium, and bonded composite resin. Fixation is maintained for 2 to 3 weeks.

When teeth with mature apices are transplanted then root canal therapy is instigated 2 to 3 weeks following transplantation. Initially
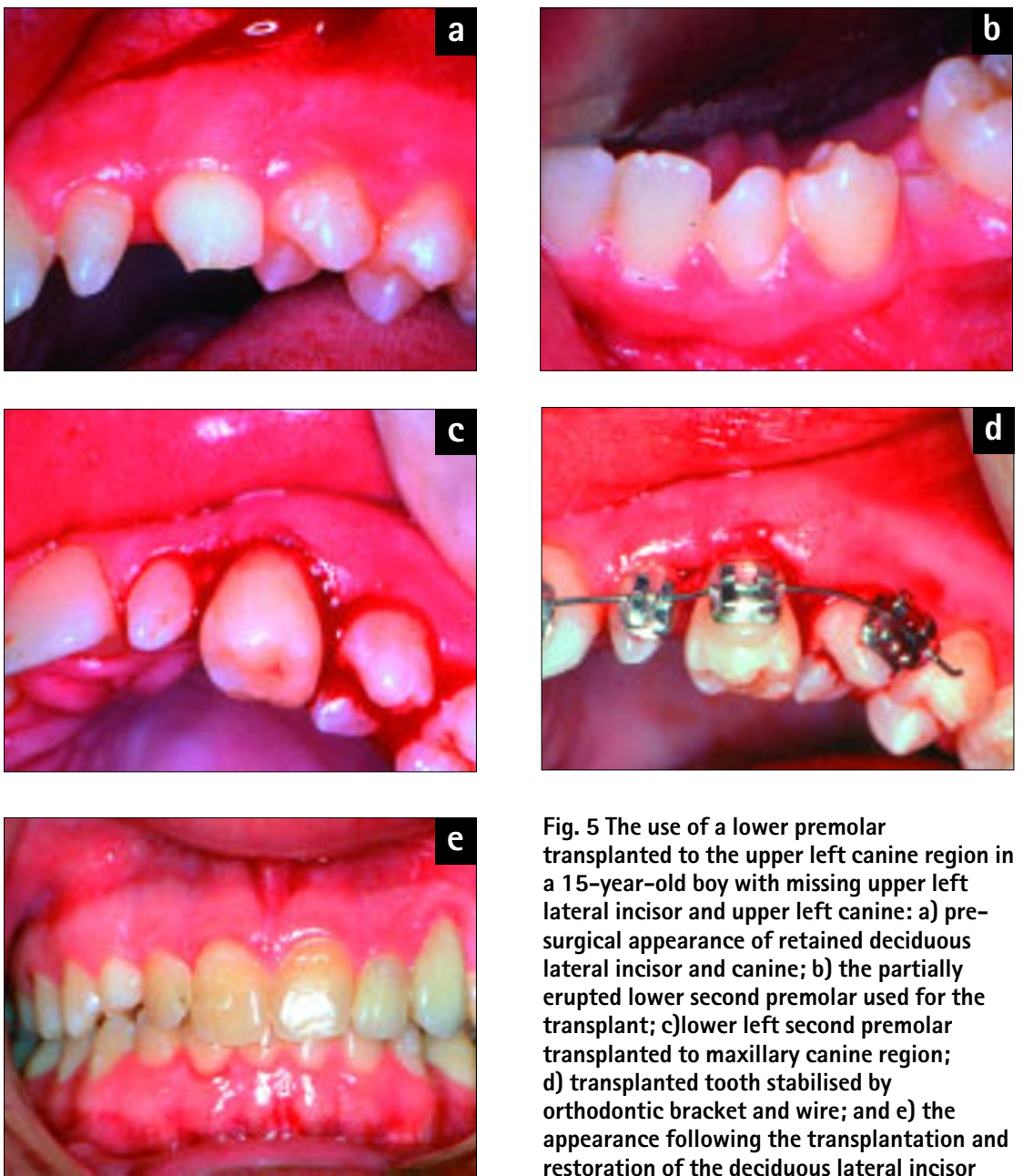

Fig. 5 The use of a lower premolar transplanted to the upper left canine region in a 15-year-old boy with missing upper left lateral incisor and upper left canine: a) presurgical appearance of retained deciduous lateral incisor and canine; b) the partially erupted lower second premolar used for the transplant; c)lower left second premolar transplanted to maxillary canine region; d) transplanted tooth stabilised by orthodontic bracket and wire; and e) the appearance following the transplantation and restoration of the deciduous lateral incisor 


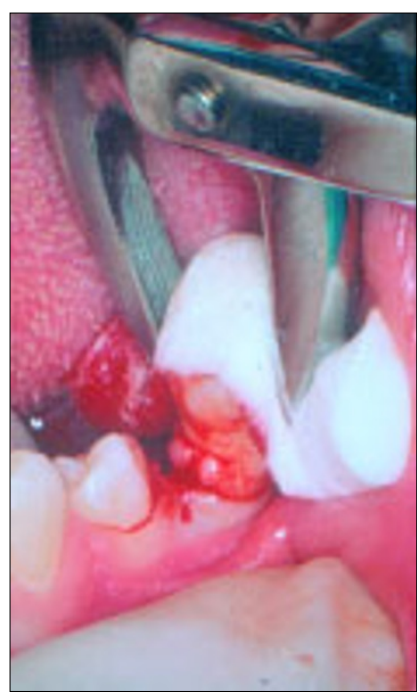

Fig. 6 The use of a cotton wool roll to protect root and crown of a tooth being used for transplantation

\section{Transplantation}

Transplantation is a viable alternative to implants in young patients with hypodontia the dressing is Ledermix or similar polyantibiotic paste followed by calcium hydroxide. The tooth is re-dressed at 3-monthly intervals, monitored and a definitive root canal filling is placed a year later, provided there is no evidence of root resorption.

The transplanted tooth can be moved orthodontically if required. This does not appear to affect further root development. ${ }^{5}$

Transplantation of teeth is a viable alternative to dental implants. Indeed, in the young patient with hypodontia transplantation has a number of advantages over implantation. These include:

- Cost (see Paper 1 in this series)

- The ability of the transplanted tooth's periodontal ligament to restore bone at the donor site, and

- The absence of disruption of bone growth.

Although the surgical aspect of transplantation is normally performed as a one stage procedure this is not essential. It is possible, with the appropriate facilities, to extract the donor tooth at the most suitable time (that is, at three-quarter root formation) and maintain the donor tooth in a cryopreservation facility until needed. ${ }^{9}$

One potential disadvantage of transplantation is failure leading to ankylosis. If an ankylosed tooth has to be removed this may lead to excessive bone destruction which may require augmentation prior to any secondary procedure, such as an implant.

\section{SURGERY AS AN AID TO PROSTHODONTICS}

In adult patients with hypodontia the provision of dental implants is an important aspect of treatment (see Parts 2 and 3 of this series). In many cases the provision of implants is not straightforward as, in addition to the lack of teeth, sufficient bone may not be available. Bony augmentation may be required and this can be provided by autogenous grafting alone or in combination with alloplastic materials and by distraction osteogenesis. The management of bony deficits is an exciting area in implantology at present and the interplay between oral surgery and tissue engineering will develop rapidly. At present the technology to model autologous bone to a desired shape at an ectopic site prior to augmentation is available, although at the experimental stage. ${ }^{10}$ Developments in this field will have major implications in the management of hypodontia in the future.

Although of great benefit to adults, dental implants are not routinely placed in children. Indeed the recommendation is that implants should not be placed until bone growth has ceased. The problems associated with the placement of implants in children include:

- Interference with bone growth

- Infra-occlusion of the implant
- Technical problems due to bone elasticity (the angle of tap may not be the angle of implant insertion)

- Maintenance

The one area where bone growth does not seem to be affected by implants is the mandible between the mental foramina. This is because the mandibular midline suture closes in the first year of life.

Studies in young animals have shown that implants do not act like erupting teeth during development but behave like ankylosed teeth. ${ }^{11}$ Studies in adolescent humans ${ }^{12}$ have shown that the degree of infra-occlusion of implants and the associated drift of the gingival margin is not directly associated with chronological age but correlates with growth in skeletal height. The more skeletal growth post-implant the greater the infra-occlusion.

Although not the routine, implants can be placed in children with hypodontia such as the child with ectodermal dysplasia. In these cases the placement of implants may be successful; the most important clinical problem being the infra-occlusion of the implants in partially dentate young patients. ${ }^{13,14}$ In edentulous young patients infra-occlusion does not seem to be a problem. In the study reported by Kearns et al. ${ }^{13}$ the placement of implants in children as young as 5 years of age did not appear to interfere with transverse or sagittal growth of the jaws. The implants moved in harmony with the growing jaws and these authors postulate that in the absence of teeth alveolar bone growth is inhibited. However, implant position is affected by sutural growth in the maxilla. These workers ${ }^{13}$ split the prosthetic bar between implants on either side of the maxillary mid-line to ensure that transverse growth of the maxilla was not affected. In some cases the mandibular bar traversing the midline was not sectioned but this did not appear to interfere with growth.

Escobar and Epker ${ }^{15}$ reported stimulation of alveolar bone growth in two edentulous children following the insertion of dental implants. These authors propose that bone growth at the alveolus is due to an increase in tension loading on the mandible without significant bending and that the stimulus for alveolar growth is the functional stress produced by mandibular flexure. Such stimulation would be produced by both teeth and implants. The early placement of implants producing functional stimulation to the jaws may also make any future surgery on the jaws simpler. ${ }^{14}$ Thus the early placement of implants in young jaws has advantages and disadvantages and further research in this area is warranted.

In some patients more major oral surgery procedures are required in the management of hypodontia. Distraction osteogenesis and osteotomies of the maxilla and or mandible may be 
necessary to obtain a satisfactory functional and aesthetic result.

\section{CONCLUSION}

Oral surgery plays a role in the management of the patient with hypodontia. The involvement of an oral surgeon in an interdisciplinary hypodontia team ensures intervention at the most appropriate time.

1. Blair G S, Hobson R S, Leggat T G. Post-treatment assessment of surgically exposed and orthodontically aligned impacted maxillary canines. Am J Orthodont 1998, 113: 329-332.

2. Hobson R S, Ledvinka J I M, Meechan J G. The effect of moisture and blood contamination on bond strength of a new orthodontic bonding material. Am J Orthodont 2001; 120: 54-57.

3. Bishara SE. Impacted maxillary canines: a review. Am J Orthodont 1992; 101: 159-171.

4. Kristerson L. Autotransplantation of human premolars. A clinical and radiographic study of 100 teeth. Int J Oral Surg 1985, 14: 200-213.

5. Kristerson L, Lagerstrom L. Autotransplantation of teeth in cases with agenesis or traumatic loss of maxillary incisors. Eur J Orthodont 1991; 13: 486- 492

6. Andreasen J 0, Andreasen F M. Textbook and Colour Atlas of Traumatic Injuries to the Teeth. 3rd edn, 1994. Copenhagen: Munksgaard, p689.

7. Andreasen J O, Paulsen H U, Yu Z, Schwartz O. A long-term study of 370 autotransplanted premolars. Part II. Tooth survival and pulp healing subsequent to transplantation. Eur J Orthodont 1990a; 12: 25-37.

8. Andreasen J O, Paulsen H U, Yu Z, Schwartz O. A long-term study of 370 autotransplanted premolars. Part III. Periodontal healing subsequent to transplantation. J Orthodont 1990b; 12: 25-37.

9. Schwartz 0. Cryopreservation as long-term storage of teeth for transplantation or replantation. Int J Oral Maxillofac Surg 1986; 15: 30-32.

10. Peter S J, Miller M J, Yasko A W, Yaszemski M J, Mikos A G. Polymer concepts in tissue engineering. Trans Acad Dent Mat 1999; 13: 17-22.

11. Odman J, Grondahl K, Lekholm U, Thilander B. The effect of osseointegrated implants on the dento-alveolar development. A clinical and radiographic study in growing pigs. Eur J Orthodont 1991: 13: 279-286.

12. Thilander B, Odman J, Grondahl K, Friberg B. Osseointegrated implants in adolescents. An alternative in replacing missing teeth? Eur J Orthodont 1994; 16:84-95.

13. Kearns G, Sharma A, Perrott D, Schmidt B, Kaban L, Vargervik K. Placement of endosseous implants in children and adolescents with hereditary ectodermal dysplasia. Oral Surg Oral Med Oral Pathol 1999; 88: 5-10.

14. Durstberger G, Celar A, Watzek G. Implant-surgical and prosthetic rehabilitation of patients with multiple dental aplasia: A clinical report. Int J Oral Maxillofac Implants 1999; 14: 417- 423.

15. Escobar V, Epker BN. Alveolar bone growth in response to endosteal implants in two patients with ectodermal dysplasia. Int J Oral Maxillofac Surg 1998; 27: 445-447. 